\title{
Fonctionnement De La Plage d'El Jadida (Côte Atlantique Marocaine) : Apport De L'étude Morphodynamique, Sédimentologique, Minéralogique Et De L'action Anthropique
}

\author{
Soukaina Khouaja \\ Mohamed Ouadia \\ El Mehdi Irzan
}

Laboratoire Géosciences et Techniques de l’Environnement,

Département de Géologie, Faculté des Sciences,

Université Chouaib Doukkalim El Jadida, Maroc

doi: 10.19044/esj.2016.v12n18p93 URL:http://dx.doi.org/10.19044/esj.2016.v12n18p93

\begin{abstract}
The multidisciplinary study of the beach of El Jadida and its sediments reflects that its shape is asymmetrical, narrow and contrasting between SE and NW which closely affects marine and wind dynamics and functioning of this beach. As profiles of beaches and the extension of the area affected by the tides, they present an argument for the elevation of the sea level had certainly warming. In addition to the Cretaceous lime stone be drock, the Quaternary calcarenites localized only in the SE richer, even more, this place in carbonate more than elsewhere in particular in winter. El Jadida beach sediments are basically homogeneous and well classified (coarse and fine) and reflect a more or less closed environment. The rocks of the SE affect the marine dynamics in rendering the texture of coarse and slightly heterogeneous sediments especially in winter but the correct classification is on the side fine sediments. The emitting provinces of minerals fuelling El Jadida Beach, via the Oued Oum Rbia and littoral drift, are essentially Jacqueline and Sidi Said Maachou El Jadida beach is under anthropogenic pressure considerable (proliferation of buildings) that promote the reduction of the area of the beach and therefore it creates a malfunction of this and an imbalance between it and marine and wind dynamics. Where urgent intervention and sound management that apply to the protection of this beach and its environment.
\end{abstract}

Keywords: El Jadida, profiles, geomorphology, mineral heavy, coastal dynamics, anthropogenic action evolution in space, changes over time 


\section{Résumé}

L’étude multidisciplinaire de la plage d'El Jadida et de ses sédiments reflète que sa forme est dissymétrique, étroite et contrastée entre SE et NW ce qui influence la dynamique marine et éolienne et le fonctionnement de cette plage. Quant aux profils de plages et de l'extension de la zone affectée par les marées, ils présentent un argument en faveur de l'élévation du niveau marin dû certainement au réchauffement climatique. En plus du substratum calcaire crétacé, les calcarénites quaternaires localisées uniquement au SE enrichissent, encore plus, cet endroit en carbonates plus qu'ailleurs en particulier en hiver. Les sédiments de la plage d'El Jadida sont en gros homogènes et bien classés (fins et grossiers) et témoignent un milieu plus ou moins fermé. Les rochers du SE influent la dynamique marine en rendant la texture des sédiments grossière et légèrement hétérogène surtout en hiver mais le bon classement est du côté sédiments fins. Les provinces émettrices des minéraux alimentant la plage d'El Jadida, via l'Oued Oum Rbia et la dérive littorale, sont essentiellement Boulaouane et Sidi Said Maachou. La plage d'El Jadida est sous pression anthropique considérable (prolifération des constructions) qui favorisent la réduction de l'espace de la plage et par conséquent elle crée un disfonctionnement de celle-ci et un déséquilibre entre elle et la dynamique marine et éolienne. D’où une intervention urgente et une gestion rationnelle s'imposent pour la protection de cette plage et de son environnement.

Mots clés : El Jadida, profils, géomorphologie, minéraux lourds, dynamique littorale, action anthropique, évolution dans l'espace, évolution dans le temps

\section{Introduction}

La plage d'El Jadida, vu sa proximité de la ville d’El Jadida et son accès facile, est une des plages marocaines les plus fréquentées par les estivants à l’échelle du Maroc. Il s’y développe des activités commerciale et culturelle pendant la période estivale. Cette plage n’a jamais fait l'objet d’aucune étude détaillée ni géomorphologique, ni minéralogique de ses sables (à l'exception de l'étude du transfert des sédiments (par Chaibi M., 2003)).

Le but de notre travail est de contribuer à mettre en exergue les caractéristiques morphologiques de cette plage et de ses profils. Il vise aussi à faire une étude sédimentologique et minéralogique de ses sables. Pour expliquer la mise en place, l'origine et l'évolution spatiotemporelle de ses dépôts, on a fait appel à une étude pluridisciplinaire.

La pression anthropique qui s’y exerce et ses conséquences sont aussi discutées dans ce travail. 


\section{Situation géographique et caractéristiques du site}

La plage d'El Jadida fait partie de la côte atlantique marocaine, elle est limitée au SE par la plage de Deauville et au NW par le port d'El Jadida.

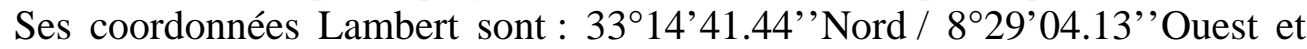
33¹5’08.90'’Nord / 8³0’03.60'’'Ouest.

Cette plage, sous forme de baie, s'étend sur une distance d'environ $1,87 \mathrm{~km}$. Elle est caractérisée au SE par un passage brutal d'une plage rocheuse à une plage sableuse (Plage d'El Jadida) (OUADIA M., 1998).Au NW, elle est plus ou moins rectiligne. Perpendiculairement à la côte, une grande partie de la plage est composée essentiellement que de l'estran. Le haut de plage et surtout la dune ne sont représentés que par endroit.

Une corniche, avec des allées, la limite du côté est. L'accès à cette plage se fait via quelques escaliers, en bois mais la plupart du temps endommagés, sinon, l'accès se fait directement (Fig. 1).

Figure 1 : Situation géographique de la plage d’El Jadida (Photo Google Earth).

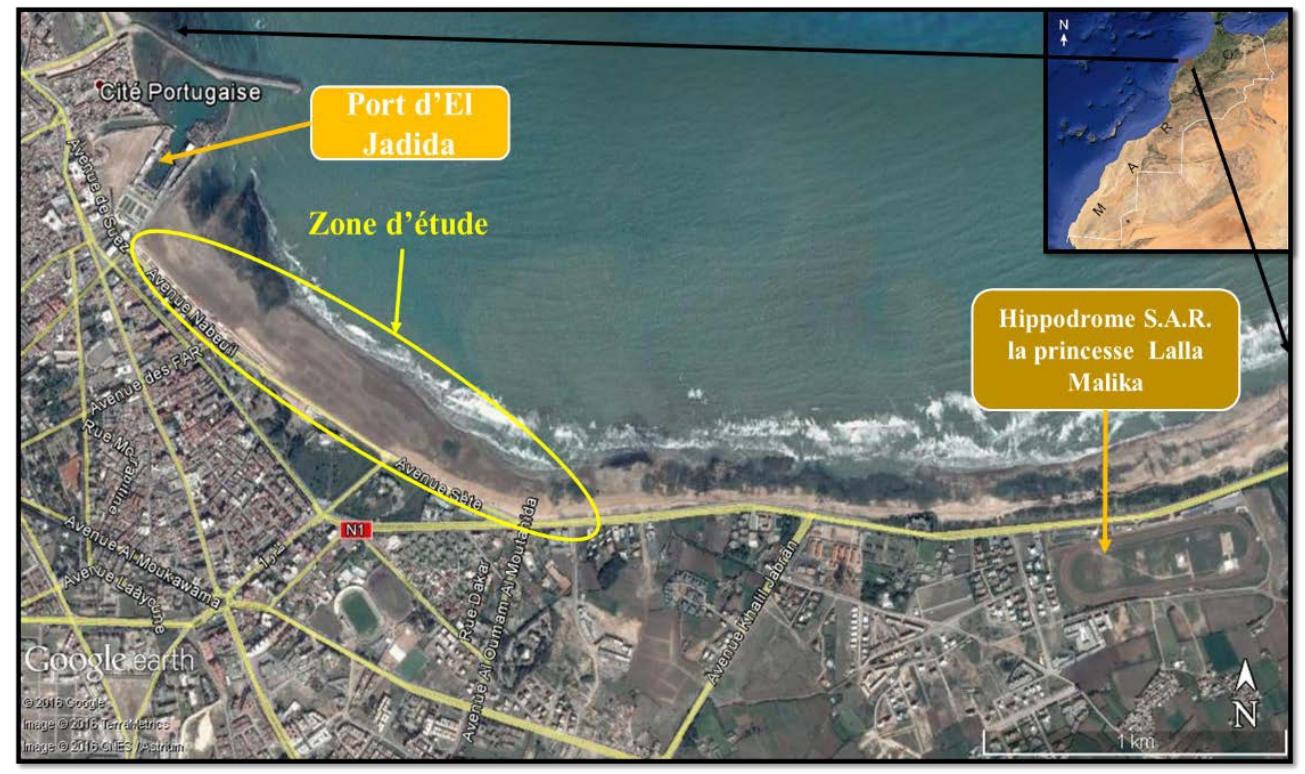

\section{Matériel et méthodes}

Dans le but d'évaluer les changements saisonniers dans cette plage, le travail de terrain a été fait en deux missions (hiver et été). Trois profils ont été levés (Nord, centre et Sud de la plage) et un échantillonnage bien réparti a été fait pour chaque mission.

Ces échantillons ont subi une analyse granulométrique selon les normes d'AFNOR (VATAN A., 1967) (fraction de taille entre 1,25 mm et $0,050 \mathrm{~mm}$ ). Avant leur analyse granulométrique, les échantillons sont attaqués par l'acide chlorhydrique puis rincés, séchés, pesés et tamisés dans une colonne de 11 tamis. Des paramètres, qui vont servir à calculer des 
indices granulométriques, ont été déduits des courbes granulométriques. Ces dernières sont tracées après le calcul des pourcentages simples et cumulés du refus de chaque tamis.

Le taux total des carbonates (après attaque des sédiments par l'acide chlorhydrique) est calculé. La détermination des minéraux lourds, précédée par leur séparation en fonction de leur densité (grâce au Bromoforme: $\mathrm{d}=2,89$ ) selon la méthode de (PARFENOFF A., et al. 1970), permet de tracer des graphiques. Ces derniers permettent de suivre l'évolution quantitative et qualitative des minéraux lourds de cette plage.

\section{Résultats}

\section{Etude géomorphologique}

Morphologie de la plage

Concernant la géomorphologie de la plage d'El Jadida, il s'agit d'une plage de forme parabolique, orientée NW-SE, dissymétrique, plus ou moins courbée au SE et relativement large au NW. Transversalement à la côte, elle est hétérogène et restreinte étant donné que rarement, et provisoirement, que la dune existe. Elle est souvent envahie par les hautes marées et les tempêtes. Seul l'estran est continu le long de la plage. Le haut de plage est discontinu et très étendu lors des périodes où la dynamique marine est calme. Dans la partie SE de la plage affleure des rochers calcarénitiques quaternaires. D’autres de même nature affleurent, par endroit, dans la zone immergée de la plage. Un déferlement des vagues est bien visible au SE et une interférence des vagues au NW. La partie NW de la plage est occupée par une corniche et des cafés.

L'évolution dans le temps de la plage d'El Jadida est déduite de la comparaison des deux photos Google Earth (2004 et 2016) prises pendant les mêmes saisons (printemps). Elle montre que la morphologie de la baie d'El Jadida est étroitement liée à la dynamique marine. Au SE, c'est la sédimentation au niveau du haut de plage qui l'emporte. Par contre, au NW, on note une sorte de compensation entre l'érosion et la sédimentation. La surface de la plage, influencée par l'action de la mer, avance d'environ 22,27 m (en douze ans : 2004-2016) témoignant d'une légère tendance à l'élévation du niveau marin (Fig. 2). 


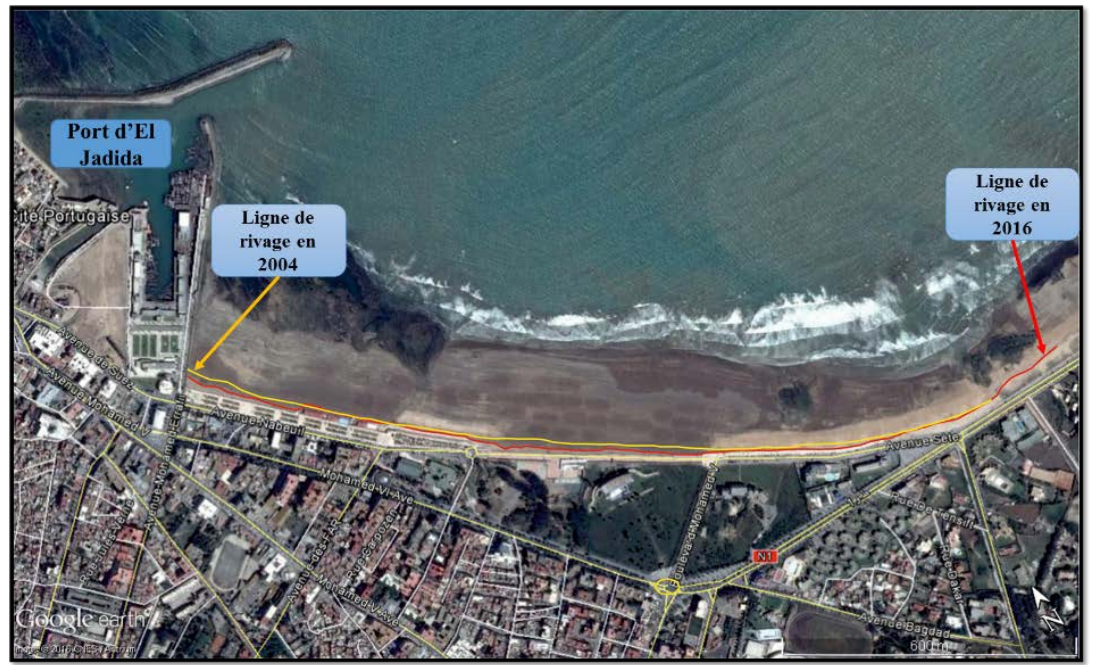

Figure 2 : Evolution de la ligne du rivage de la plage d’El Jadida entre 2004 et 2016 (photos Google Earth).

\section{Profils de la plage}

L'étude des profils de la plage d'El Jadida est faite pour la première fois (à l'exception de l'étude des profils de plage dans la zone avoisinante « Haouzia » faite par Chaibi M, (2003). Les profils de cette plage, sont hétérogènes et montrant une variation dans l'espace et dans le temps. Ils sont relativement plus amples aux extrémités de la plage NW et SE et à son centre. L'écart, le plus important, de l'altitude de ces derniers est celui du profil 7 au centre de la plage en face de la déviation de la corniche. La berme est plus individualisée en hiver surtout pour le secteur centre (profil 8). En été, l'accrétion est plus remarquable surtout au niveau du haut de plage, en particulier au SE (profil 10) (Fig. 3) (Fig. 4).

Figure 3 : Localisation des profils étudiés de la plage d'El Jadida.

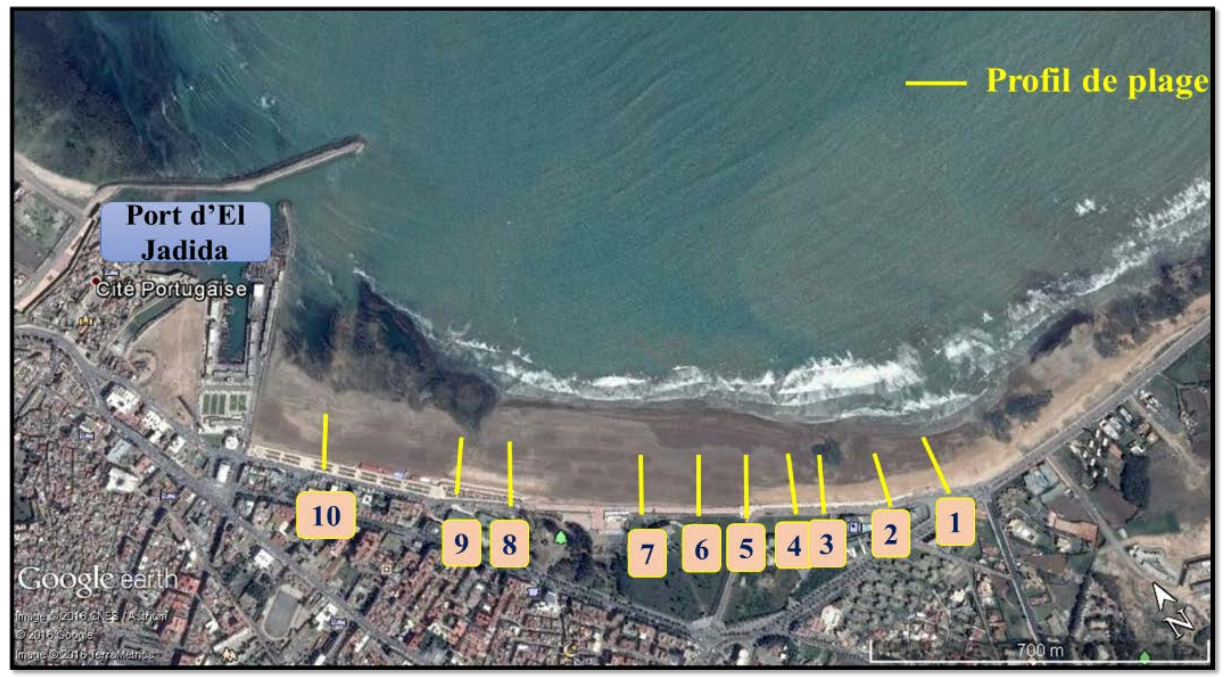




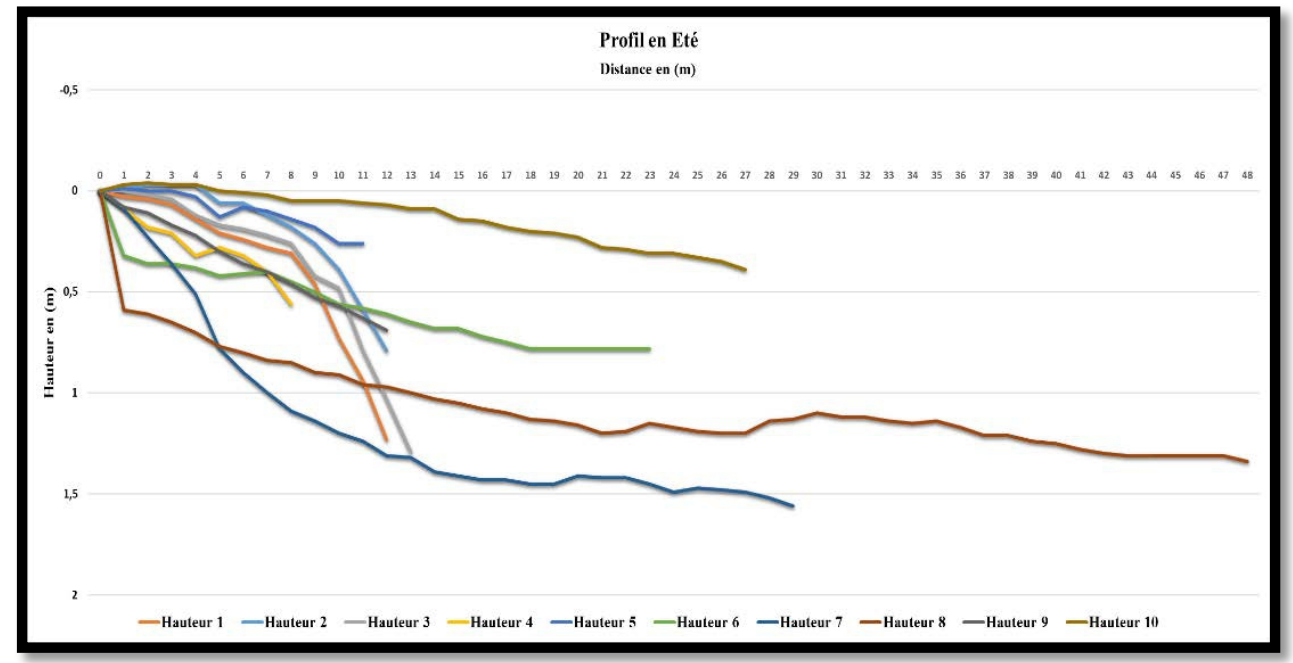

Figure 4 : Evolution des profils de la plage d’El Jadida en Eté 2015.

\section{Etude sédimentologique}

\section{Calcimétrie}

Le taux des carbonates est variable et peut atteindre $90 \%$ au SE où il est plus élevévis-à-vis le NW. Concernant la variation saisonnière, ce dernier est légèrement élevé en hiver plus qu'en été. Cependant, perpendiculairement à la plage, c'est dans le haut de plage où est enregistré le taux des carbonates le plus important par rapport à l'estran (Fig 5).

Figure 5 : Evolution des taux des carbonates dans les sables de la plage d'El Jadida (EL : El Jadida ; Es : estran ; HP : haut de plage ; D : dune)

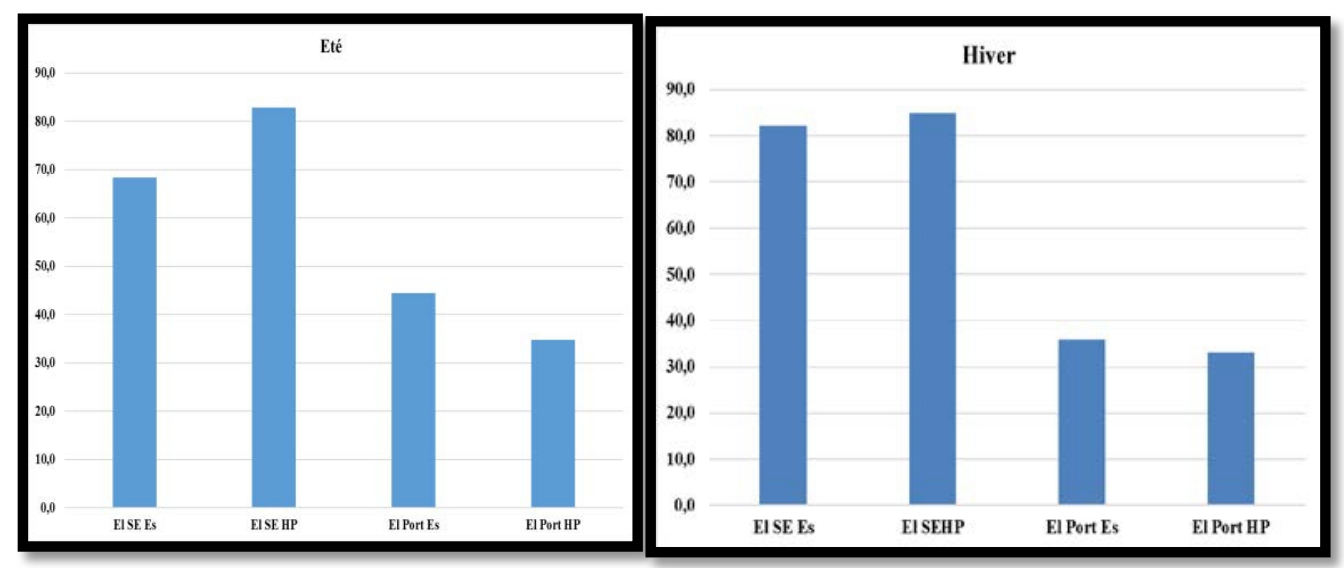




\section{Granulométrie}

\section{Courbes granulométriques}

La majorité des courbes granulométriques sont unimodales. La seule courbe bimodale est celle des sédiments de l'estran au SE. L'unique mode pour les échantillons est au niveau des sédiments grossiers pour la partie SE et fins pour la partie NW. Perpendiculairement à la plage, l'unique mode des courbes granulométriques tend à être au niveau des sédiments grossiers dans le haut de plage par rapport à l'estran. En fonction des saisons, c'est à l'hiver que l'unique mode est du côté des sédiments grossiers par rapport à l'été (Fig. 6).

Figure 6 : Evolution des modes des sables de la plage d'El Jadida.
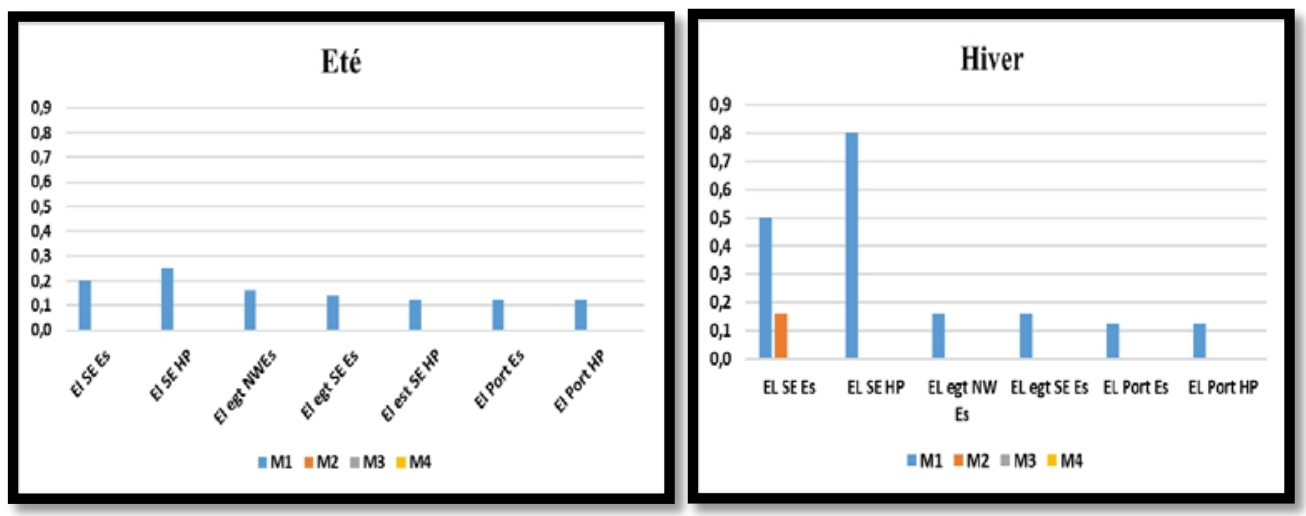

\section{$>\quad$ Paramètres granulométriques}

Les valeurs des paramètres granulométriques sont très contrastées et très élevées au SE plus qu'au NW. Perpendiculairement à la plage, la valeur des paramètres granulométriques des sédiments du haut de la plage est plus élevée que celle de l'estran. En fonction des saisons, c'est à l'hiver où la valeur des paramètres granulométriques est élevée par comparaison à celle

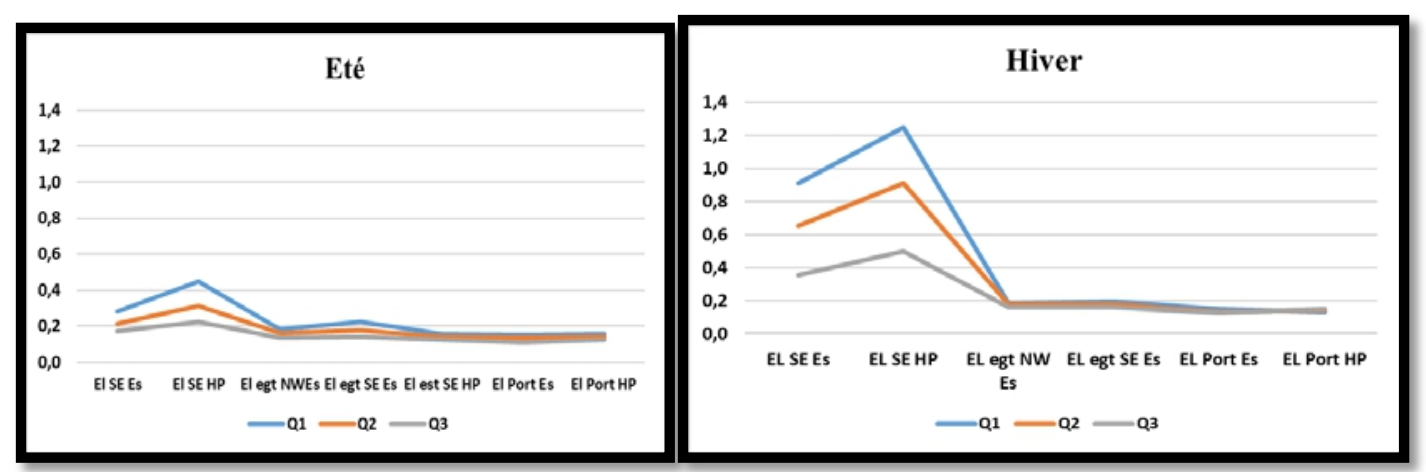

l'été (Fig. 7).

Figure 7 : Evolution des paramètres granulométriques (Q1, Q2 et Q3). 


\section{$>\quad$ Indices granulométries}

- $\quad$ Sorting index (So)

So est, en gros, compris entre 1,3 et 1,6. Du SE au NW, les valeurs de So diminuent. Transversalement à la plage, on ne remarque pas trop de changement. Quant aux variations saisonnières, les valeurs de So de l'hiver sont plus importantes par rapport à celle de l'été.

\section{- Skewness(Sk)}

SK est généralement au tour de 1. Du NW au SE, les valeurs de Sk diminuent légèrement. Perpendiculairement à la plage, les valeurs de Sk diminuent relativement en allant de l'estran vers le haut de plage. Les valeurs de Sk montrent une nette diminution en passant de l'hiver à l'été (Fig. 8).
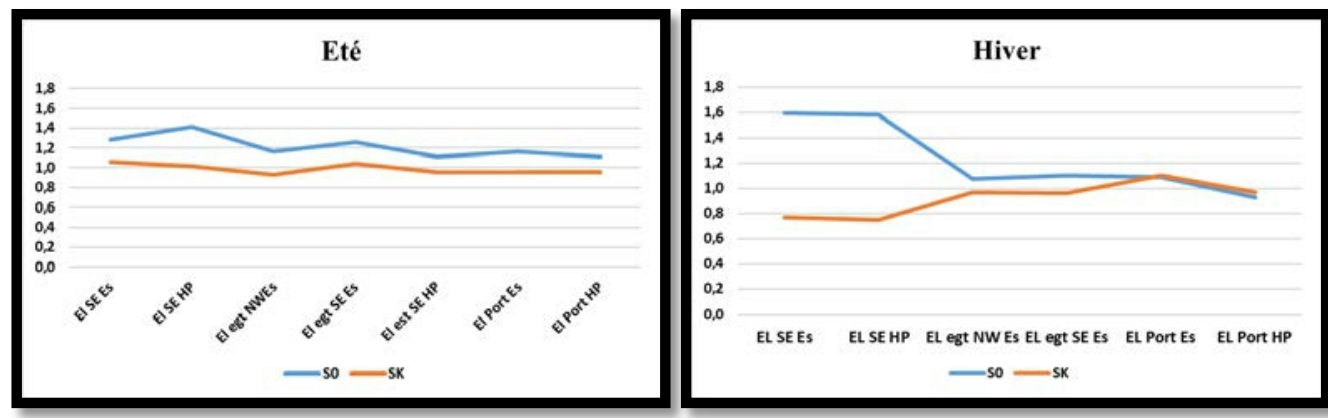

Figure 8 : Evolution des indices granulométriques (So et Sk).

\section{Etude minéralogique (minéraux lourds)}

Les minéraux lourds des sédiments de la plage d’El Jadida présentent un cortège minéralogique varié de point de vue quantité et qualité. Le pyroxène, le disthène et les micas ainsi que le zircon prédominent nettement les autres minéraux. Une tendance à la diminution du taux des micas et du disthène est remarquable du SE au NW et du disthène de l'estran vers le haut de plage (contrairement au pyroxène) (Fig. 9).

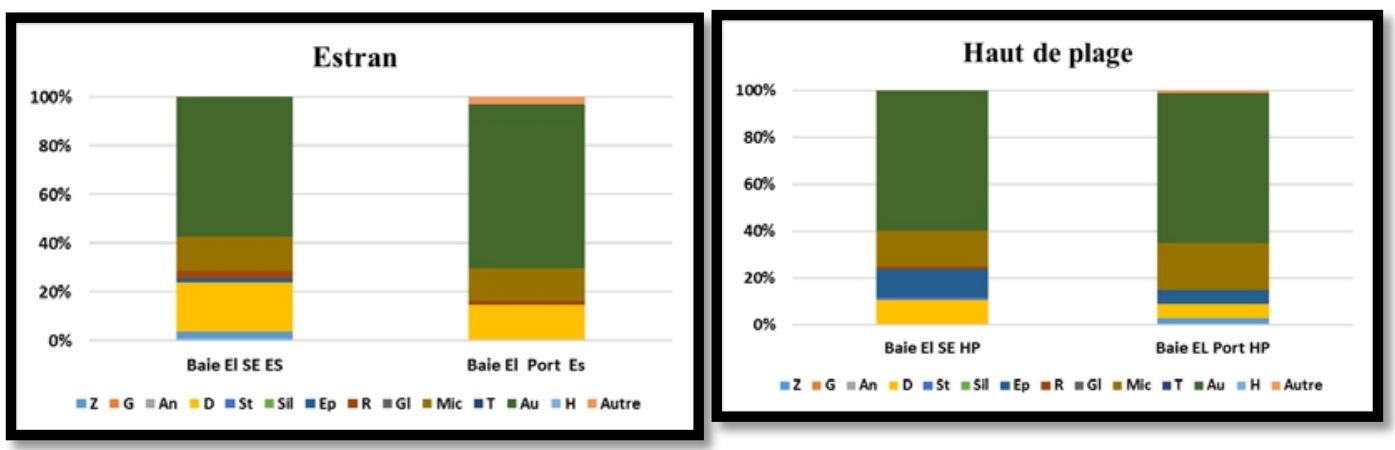

Figure 9 : Evolution du cortège des minéraux lourds des sédiments de la plage d’El Jadida. 


\section{Action anthropique}

La plage d'El Jadida est fortement exploitée et rétrécie à cause des constructions commerciales (surtout des cafés) et des douches qui sont bâties dans la zone correspondant, complètement au haut de la plage et partiellement à son estran au NW.

De multiples canaux d'eau de pluies (plusieurs secondaires et un principal) déversent directement dans la plage. Des escaliers, en bois ou en béton, mal conçus et mal repartis sur la plage sont fréquents (Fig. 10).

Figure 10 : Action anthropique

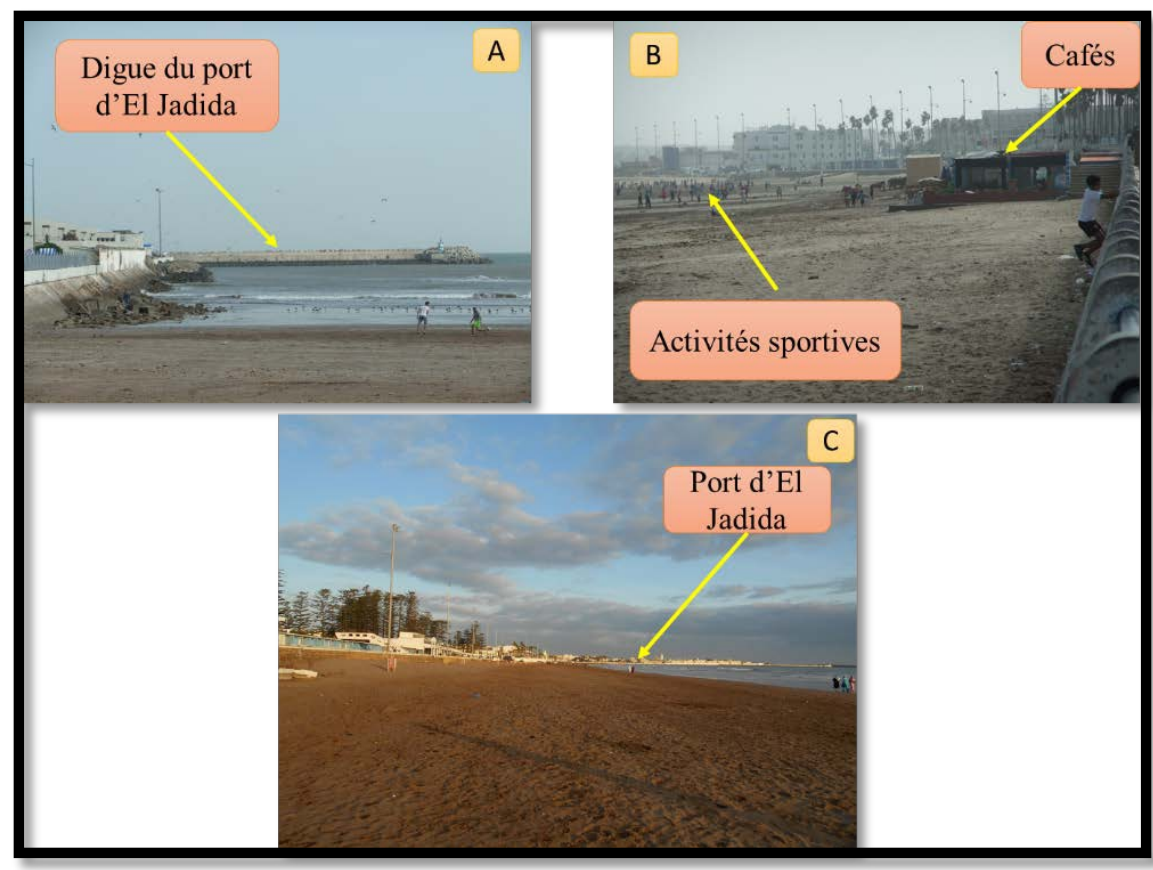

\section{Interprétation et discussion}

\section{Etude géomorphologique}

\section{Morphologie de la plage}

La plage d'El Jadida est hétérogène de point de vue forme, orientation et symétrie. Elle est très restreinte au NW et large au SE. La dune est presque absente et ne parait qu'occasionnellement. La pente de la plage est relativement forte au SE par rapport au NW.

Lors des tempêtes, toute la plage est envahie par les marées. L'extrémité SE se distingue par l'affleurement de quelques rochers calcarénitiques. Le contraste entre la moitié nord et la moitié sud de la plage s'amplifie à cause de la quantité importante d'eau de pluie qui se jette dans la plage au niveau du canal principal en causant un ravin profond au centre de la plage. 
Dans la partie SE de la plage affleurent des rochers calcarénitiques quaternaires. D'autres de même nature affleurent, par endroit, dans la zone immergée de la plage. Un déferlement des vagues est bien visible au SE et une interférence des vagues au NW. La partie NW de la plage est occupée par une corniche.

Dans le temps, une légère tendance à l'extension de la zone affectée par les marées est mise en évidence en comparant les photos Google Earth (2004-2016). Elle témoigne d'une élévation du niveau marin en relation, sans doute, avec le réchauffement climatique.

\section{Profils}

L’hétérogénéité des profils de plage signifie l'action de plusieurs facteurs dont, principalement, les dynamiques marine et éolienne et l'étroitesse ainsi que la dissymétrie de la plage.

\section{Etude sédimentologique}

Jusqu'à maintenant, cette plage n'a pas été traitée de point de vue sédimentologique, d'une manière très détaillée si on la compare aux autres régions littorales atlantiques marocaines étudiées par (ABOUMARIA K., 1993 ; CHAIBI M., 2003 et CHAIBI et SABATIER F., 2010; ABERKAN M.et al., 2014 et 2015 et OUADIA M. et al., 2015).

\section{Calcimétrie}

Le taux des carbonates élevé au SE témoigne qu'en plus du substratum calcaire crétacé, les rochers calcarénitiques (KHOUAJA S. et al., 2016) et les colonies de coquilles à test calcaire qui y se fixent participant à l'alimentation des sédiments de la plage en carbonates. Ce taux plus élevé à ce niveau mais aussi en hiver pour toute la plage s'explique par la dynamique forte qui érode d'avantage ces rochers et le substratum en hiver.

\section{Granulométrie}

$>\quad$ Courbes granulométriques (Simples et cumulées)

L'unimodalité des courbes granulométriques de la majorité des sédiments reflète l'homogénéité des sédiments et par conséquent un milieu plus ou moins fermé.

La légère hétérogénéité des sédiments au SE est du certainement à l'agitation et aux turbulences causées par les rochers du SE.En fonction des saisons, c'est à l'hiver que l'unique mode est du côté des sédiments grossiers par rapport à l'été reflétant que la dynamique marine commence à se calmer pendant l'été. 


\section{$>\quad$ Paramètres granulométriques}

Les valeurs des paramètres granulométriques élevées au SE plus qu'au NW témoignent d'une dynamique forte au SE plus qu'au NW à cause vraisemblablement de l'action de l'agitation favorisée par les rochers. Perpendiculairement à la plage, la valeur des paramètres granulométriques des sédiments du haut de la plage est plus élevée que celles de l'estran à cause d'un brassage fréquent des sédiments par l'action anthropique. En fonction des saisons, c'est à l'hiver où la valeur des paramètres granulométriques est élevée par comparaison à celle l'été à cause des tempêtes fortes pendant l'hiver.

\section{$>\quad$ Indices granulométriques}

\section{- $\quad$ Sorting index (So)}

Les valeurs, en général, faibles de So prouvent des sédiments bien classés. Au NW, les valeurs, légèrement, élevées de So témoignent d'un mauvais tri relatif des sédiments à cet endroit. Ce mauvais tri est également remarqué dans les sédiments de l'hiver à cause d'un brassage fort des sédiments par des tempêtes.

\section{- $\quad$ Skwness (Sk)}

Les valeurs de SK qui sont en gros au tour de 1 prouvent que le classement intéresse la fraction moyenne des sédiments. Du SE au NW, les valeurs de Sk augmentent légèrement et témoignent que le classement est du côté de sédiments grossiers. Perpendiculairement à la plage, les valeurs de Sk diminuent relativement en allant de l'estran vers le haut de plage où le classement est du côté des sédiments fins. Les valeurs de Sk montrent une nette diminution en passant de l'hiver à l'été ce qui reflète que le classement des sédiments est du côté sédiments fins en été.

\section{Etude minéralogique (minéraux lourds)}

Le cortège des minéraux lourds des sédiments de la plage d’El Jadida est varié quantitativement et qualitativement.

Il est dominé par le pyroxène, le disthène et les micas témoignant que malgré la diversité des sources émettrices des minéraux, les terrains métamorphiques et magmatiques restent les plus productifs de minéraux alimentant la plage d'El Jadida. Les terrains métamorphiques sont représentés par les formations paléozoïques de Boulaouane et magmatiques par les basaltes permo-triasiques de Sidi Said Maachou. Ces minéraux sont acheminés par l'Oued Oum Rbia jusqu'à son embouchure à Azemmour puis repris et distribué par la dérive littorale le long de la plage d’El Jadida (Fig 11). 


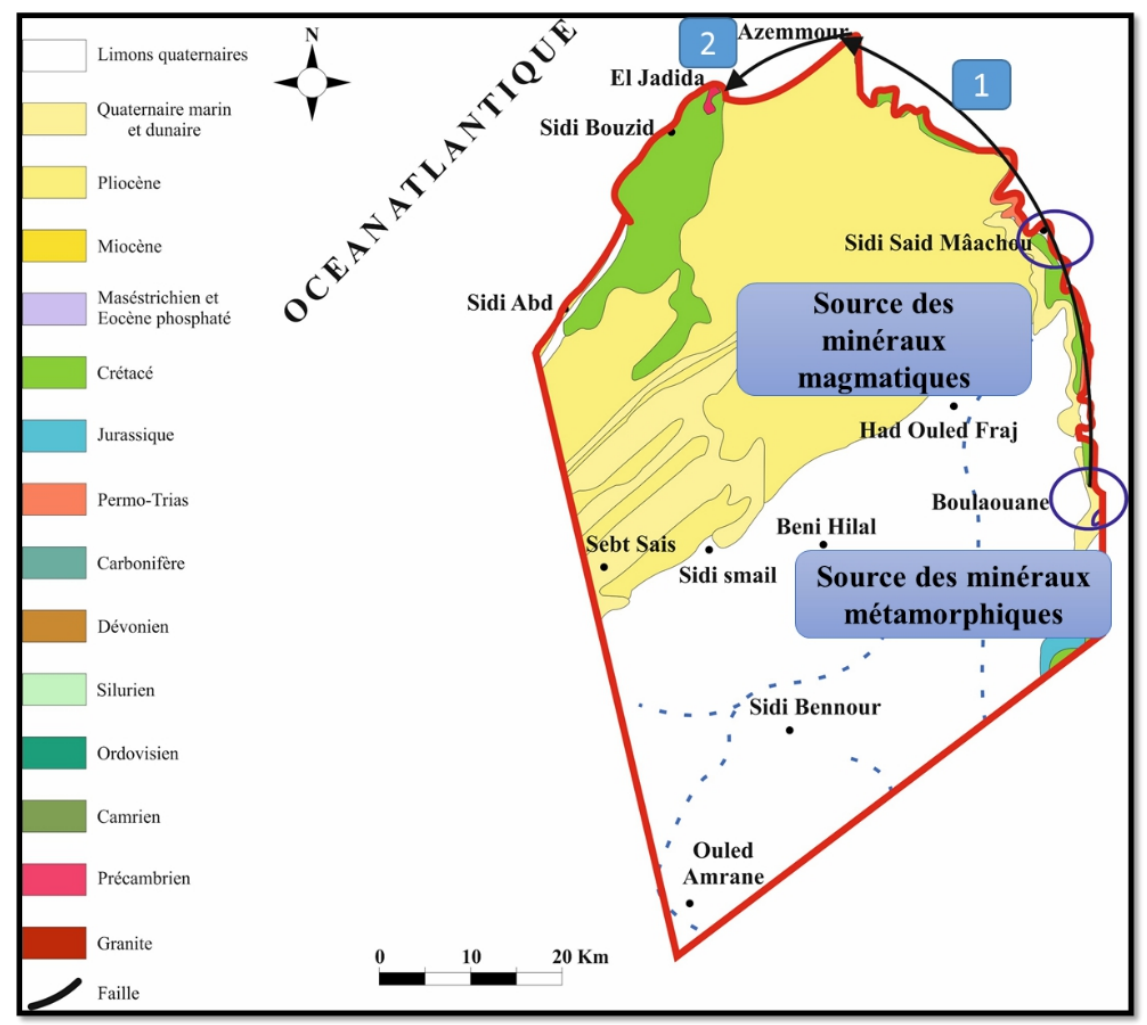

Figure 11 : Origine et transite des minéraux lourds (1: via Oued Oum Rbia ; 2 : par la dérive littorale).

\section{Action anthropique}

La plage d'El Jadida a subi, et continu à subir, une action anthropique. Elle peut être résumée, essentiellement, dans l'activité touristique (prolifération des cafés et des douches). Ces constructions sont bâties aux dépens de la plage qui devient dépourvue complètement de sa dune et en grande partie de son haut de plage. Par conséquent la plage est devenue très rétrécie ce qui gêne la dynamique marine et son interaction avec la plage. A ceci peut s'associer une infrastructure fragile et une gestion inadéquate de la dite plage.

De multiples canaux d'eau de pluies (plusieurs secondaires et un principal) déversent directement dans la plage. Des escaliers, en bois ou en béton, mal conçus et mal repartis sur la plage sont fréquents. D’autres plages avoisinantes ont connu également une action anthropique (OUADIA M. et ABERKAN M., 2003 et OUADIA M. et al., 2005).

\section{Conclusion}

La forme de la plage d'El Jadida n'est pas homogène de point de vue étendue, symétrie et orientation. Sa partie SE est relativement large, à pente 
forte et à rochers par rapport au NW ce qui entraine un fonctionnement contrasté de celle-ci entre SE et NW. La dune, lorsqu'elle existe occasionnellement là, est limitée et discontinue dans l'espace. L'espace assez restreint de la plage ne laisse pas la dynamique marine et éolienne fonctionner naturellement.

Quant aux profils de plages, leur longueur faible révèle la surface restreinte de la plage.

Le taux des carbonates élevé au SE témoigne de l’influence directe des rochers calcarénitiques qui aliment, en plus des calcaires du crétacé, les sédiments de la plage. Cette alimentation est proportionnelle au degré de l'érosion de ces rochers d'où l'importance des carbonates en hiver à cause des tempêtes et de l'agitation de la mer.

Les sédiments de la plage d'El Jadida sont en gros homogènes (courbes granulométriques simples unimodales) et témoignent un milieu plus ou moins fermé. La légère hétérogénéité et des sédiments et leur texture grossière au SE et en hiver est due respectivement à l'agitation de cet endroit due à l'action des rochers et aux tempêtes de l'hiver.

Les valeurs de So et de Sk prouvent que les sédiments (grossiers et fins) sont, en gros, bien classés mais au SE, le classement est plutôt du côté des sédiments fins.

L'abondance de minéraux métamorphiques et magmatiques témoigne de la participation des quartzites de Boulaouane et des basaltes de Sidi Said Maachou à l'alimentation de la plage d'El Jadida par l'intermédiaire de l'Oued Oum Rbia et par la dérive littorale.

Dans la plage d'El Jadida, la pression anthropique (prolifération des constructions) est très remarquable. Elle participe à la réduction de la surface de la plage et par conséquent elle crée un déséquilibre entre ce milieu et la dynamique marine et éolienne.

D’où la nécessité d'une intervention et l'amélioration de la gestion pour une meilleur protection de cette plage et de son environnement.

\section{References:}

ABERKAN M., ABOUMARIA Kh. et OUADIA M., (2014) - Apport de l'observation du paysage côtier actuel et de l'organisation des séquences littorales sur la paléogéographie du Pléistocène récent au Nord-Ouest du Maroc. Actes RQM6, Tanger, pp. 79-85

ABERKAN M., ABOUMARIA Kh., ZAGHLOUL M.N. et OUADIA M., (2015) - Dynamique littorale et instabilité des formations quaternaires de la façade atlantique marocaine : Impact sur l'aménagement de la zone costière entre Rabat et Larache (Maroc). RQM8, Nador, pp. 39-40. 
ABOUMARIA K., 1993. Les formations quaternaires du Sahel des Doukkala (Meseta occidentale marocaine) : mise en place et évolution post-sédimentaire. Thèse Doctorat 3ème cycle, Univ. Mohammed V, Fac. Sci., Rabat, 186 p.

CHAIBI M., 2003. Dynamique sédimentaire et morphogenèse actuelle du littoral d'El Jadida (Maroc). Thèse Doctorat Es-sciences, Univ. AixMarseille, 235 p.

CHAIBI M. et Sabatier F., 2010Budget sédimentaire du littoral de la baie d'El Jadida (Maroc). Méditerranée, 115 | 2010 : Rivages p. 116-124méditerranéens. KHOUAJA S., OUADIA M., MOURABITI I. et IRZAN E., (2016) L'érosion de la falaise de Sidi Bouzid (Côte atlantique marocaine) : Quantification et évolution spatio-temporelle. ESJ, Vol. 12, No 1, pp. 150163.

OUADIA M.,(1998) « Les formations plioquaternaires dans le domaine mésétien occidental ente Casablanca et Safi : Géomorphologie, Sédimentologie, Paléoenvironnements quaternaires et Evolution actuelle » Thèse Doct. d'Etat, Univ. Mohammed V, Rabat, Maroc 319 p.

OUADIA M. et ABERKAN M. (2003) - Influence des actions naturelle et anthropique sur la Méséta occidentale marocaine (entre El Jadida et Safi). Actes du colloque : Les $2^{\text {ème }}$ journées des Géosciences de l'Environnement, Kénitra, pp. 1-7.

OUADIA M., ABERKAN M., ABOUMARIA Kh. et NISSOUL A. (2005) Action anthropique dans la Méséta côtière marocaine (Entre Casablanca et Safi) : depuis le Pléistocène supérieur jusqu'à l'Actuel., 3ème Rencontre des quaternaristesmarocains, Marrakech, p. 47.

OUADIA M., ABERKAN M. et ABOUMARIA K. (2015) - Le complexe dunaire quaternaire du JorfLasfar (Meseta côtière occidentale marocaine) : lithostratigraphie, sédimentologie et néotectonique. Paralia, Vol. 8, $\mathrm{N}^{\circ}$. 1, pp n02.1-n02.14, DOI: http://dx.doi.org/10.5150/revue-paralia.2015.n02

PARFENOFF A., POMEROL C. et TOURENG J. (1970) - Les minéraux en grains : méthodes d'étude et détermination. Masson et çie Ed., Paris, 499 p. VATAN A. (1967) Manuel de sédimontologie .TechnipEd . Paris 397 p. 